\title{
Emploi du vaccin lanoliné antipéripneumonique en milieu sain. Valeur antigénique
}

\author{
par R. TESTU
}

LE'vaccin utilisé est constitué par de la sérosité pleurale enrobée dans un mélange de lanoline et d'huile d'arachide.

Lanoline $\ldots \ldots \ldots \ldots \ldots \ldots \ldots \ldots, \quad 5 \mathrm{gr}$.
Sérosité pleurale $\ldots \ldots \ldots \ldots \ldots$
Huile d'arachide stérilisée........

Le troupeau de bœeufs producteurs de sérum du centre est immunisé dans les premiers jours du mois d'Octobre (2 cc. vaccin par animal).

Réaction vaccinale locale, débutant trente-six à quarante-huit heures après l'injection sous-cutanée. L'œdème se développe dans lès trois à quatre jours qui suivent, puis reste stationnaire. Vers le neuvième ou le dixième jour, on note une résorption lente de l'celème avec induration persistante qui, deux mois après, a la grosseur d'une noix (il est visible quatorze mois plus tard sur la plupart des sujets vaccinés).

Réaction identique observée sur les animaux du 'troupeau du village de Boghé, vaccinés en Novembre: A la date du 20 Février 1944, aucun cas de péripneumonie ne s'est encore déclaré dans ces troupeaux.

Ne pouvant encore conclure à l'efficacité ou à l'inefficacité du vaccin, il était nécessaire d'éprouver les sujets vaccinés (contamination expérimentale ou directe).

Sept sujets (marqués des lettres XE et numérotés de 1 à 7) ayant reçu une dose vaccinale début Octobre, reçoivent, cinq mois plus tard, 5 cc. de sérosité pleurale et puImonaire dans le tissu conjonctif sous-cutané en arrière de l'épaule.

De même six autres sujets vaccinés à la même date (marqués des lettres XCH et numérotés de I à 6) sont inoculés au chanfrein ( $4 \mathrm{cc}$. de sérosité virulente).

Un bouvillon non vacciné est inoculé comme témoin.

Ces quatorze sujets sont des bouvillons de 2 ans $1 / 2$ à 3 ans $1 / 2$ choisis tels afin que les résultats de l'expérience ne soient pas faussés par une atteinte antérieure toujours possible en zone d'endémicité.

Les treize sujets inoculés font une. légère hyperthermie, puis tout rentre dans l'ordre.

La réaction locale est assez légère sur dix d'entre eux, et régresse rapidement quatre à cinq jours après son apparition; par contre; sur les trois autres, l'engorgement est plus accusé mais sans prendre toutefois une allure envahissante. Il persistera une dizaine de jours.
Ces trejze sujets sont suivis du 6 Février au 10 Avril. Seul, le $n^{\circ}$ XI fait une poussée thermique pendant. cinq jours. L'animal tousse; appétit nul et rumination irrégulière. A partir du 4 Mars la température s'abaisse et, le 7 Mars, la mort survient en hypothermie.

Signes nécropsiques. - L'autopsie révèle une péripneumonie "classique : lésions bilatérales, adhérences de la plèvre, surabondance de liquide pleurétique, avec nombreuses membranes.

Le bouvillon témoin succombe le 9 Mars, mais sans avoir présenté de signe apparent. Congestion pulmonaire avec œdème. Aucune lésion peripneumonique.

Cette première expérience pourrait faire conclure à l'efficacité réelle du vaccin lanoliné : douze sujets sur treize résistent à une dose virulente de sérosité pleurale et pulmonaire.

Néanmoins, nous devons faire la part et de la résistance naturelle des jeunes et de nombreux facteurs mal connus à ce jour. Ces réserves s'imposent.

En Juin suivant, la péripneumonie se déclare, une fois de plus; dans le troupeau du Centre sérumigène. La totalité de l'effectif avait été pourtant vaccinée huit móis plus tôt. De Juin à Octobre nous observons de nombreux cas typiques dont onze morts : quatre bœufs producteurs de sérum, sept bouvillons réfractaires à la peste bovine.

Lésions de péripneumonie classique (bilatérales en majorité). Ces résultats montrent la réceptivité des sujets vaccinés après un délai de sept à huit mois.

Conclusions. - Lès observations faites permettent de formuler les conclusions suivantes :

a) innocuité absolue du vaccin lanoliné en milieu indemne;

b) il semble 'qu'il $\mathrm{y}$ ait immunité active après vaccination;

c) cetle immunité serait assez lenie à s'établir (un mois et demi à deux mois);

d) l'immunité conférée est de courte durée (inférieure à sept mois);

e) la vaccination préventive n'atténue pas les réactions consécutives à une nouvelle contamination naturelle au-delà de la limite d'efficacité du vaccin. 